\title{
Important Roles of Health Professionals in Maternal HIV Disclosure Among HIV-Infected Women in China
}

\author{
Qian Wang ${ }^{1} \cdot$ Zixin Wang ${ }^{2,3} \cdot$ Xiaomeng Ma ${ }^{4} \cdot$ Yuan Fang ${ }^{2} \cdot$ Phoenix K. H. Mo ${ }^{2,3}$ (D) Joseph T. F. Lau ${ }^{2,3}$
}

Published online: 21 June 2019

๑) Springer Science+Business Media, LLC, part of Springer Nature 2019

\begin{abstract}
Maternal HIV disclosure to children is beneficial for both mothers and children. A cross-sectional survey was conducted to investigate the prevalence of maternal HIV disclosure to at least one living child aged $>5$ years among 292 HIV-infected mothers in Guangxi Province, China. Among all participants, $45.2 \%$ had self-disclosed their HIV positive sero-status to at least one living child aged $>5$ years. After adjusting for the significant background variables, participants self-reported health professionals in governmental antenatal care clinics had discussed with them about maternal HIV disclosure (adjusted odds ratios, aOR: 5.85), had received counseling services (aOR: 7.84) or support (aOR: 8.75) from these health professionals when making decision on maternal HIV, and perceived higher empathy of these health professionals (aOR: 1.09) were more likely to have reported maternal HIV disclosure. Instrumental and affective interactions between health professionals and HIV-infected mothers were important facilitators of maternal HIV disclosure.
\end{abstract}

Keywords Maternal HIV disclosure to children $\cdot \mathrm{HIV}$-infected mothers $\cdot$ Doctor-patient interactions $\cdot$ China

\section{Introduction}

With antiretroviral therapy (ART), most of HIV-infected women can live healthy lives and continue to play important roles as mothers. Self-disclosure is a process that involves

Qian Wang and Zixin Wang have contributed equally to this manuscript.

Electronic supplementary material The online version of this article (https://doi.org/10.1007/s10461-019-02566-w) contains supplementary material, which is available to authorized users.

Phoenix K. H. Mo

phoenix.mo@cuhk.edu.hk

Joseph T. F. Lau

jlau@cuhk.edu.hk

1 National Center for Women and Children's Health, China Center for Disease Control and Prevention, Beijing, China

2 Centre for Health Behaviours Research, JC School of Public Health and Primary Care, The Chinese University of Hong Kong, Hong Kong SAR, China

3 Shenzhen Research Institute, The Chinese University of Hong Kong, Shenzhen, China

4 Department of Health Policy and Management, John Hopkins Bloomberg School of Public Health, Baltimore, MD, USA self-initiated telling of previously concealed and potentially stigmatizing information to one or more persons with the intention of improving one's sense of psychological wellbeing and/or maintaining relationship [1]. Maternal HIV disclosure to children is beneficial for both mothers and children [2]. Numerous studies showed that maternal HIV selfdisclosure had benefits for HIV-infected mothers in terms of better mental health status, higher adherence to ART, and improved family relationship and support [3-14]. Ensuring timely, age-appropriate maternal HIV disclosure could also increase quality of custody and emergency care planning for children [7, 15]. Similarly, many studies observed improvement in emotional and social functioning, feeling better prepared for the future, closer to their HIV-infected mothers and less afraid among children following maternal HIV self-disclosure $[6,8,14,16,17]$. For older children, maternal HIV self-disclosure was associated with more involvement in family decision-making and assisting the mother with her illness [14]. Therefore, the World Health Organization (WHO) recommends children of school age should be informed about their parents' HIV status, and younger children should be informed gradually, in order to accommodating their cognitive skills and emotional maturity [18].

Across countries, low maternal HIV disclosure to children was a potential threat to HIV-infected mothers $[19,20]$. 
Previous studies showed that the prevalence of maternal HIV disclosure was only $39.0 \%$ in China, $20-67 \%$ in the U.S., and $11-50 \%$ in other countries [21-25]. Population size of HIV-infected mothers is very large in China (over 200,000 in 2011) [26]. Traditional Chinese culture always does not encourage disclosure of stressful events such as maternal HIV infection [27]. HIV-infected mothers in China often find it difficult for maternal HIV disclosure due to various concerns $[13,24]$. They may struggle about when and how to disclose. Many of them have low level of confidence to talk about HIV or to explain the cause of their illness with their children [13, 24].

Health professionals providing prevention of mother-tochild transmission (PMTCT) services play important roles in facilitating maternal HIV disclosure. Studies showed that PMTCT service utilization was associated with higher maternal HIV disclosure [28, 29]. A pilot randomized controlled trial targeting people living with HIV (PLWH) in China showed that intervention delivered by health professionals was effective in facilitating parental HIV disclosure decision making [30]. In the Chinese cities (Dehong, Qinzhou and Hezhou) where this study was conducted, governmental antenatal care (ANC) clinics are providing PMTCT services, HIV treatment and care for HIV-infected women. A HIV-infected woman is followed up by a same ANC health professional (i.e. case manager) since diagnosis. This health professional will be responsible for providing all relevant PMTCT, HIV treatment or care services to her. The professional-client interaction theory was used to understand how interaction between health professionals in these ANC clinics and HIV-positive mothers influence maternal HIV disclosure in this study [31]. According to the theory, doctor-patient interactions can be divided into instrumental interaction and affective interaction [31]. Instrumental interactions refer to behaviors that address patients' health needs or problems [31]. In this study, instrumental interaction included initiating discussion, and provision of counseling and supports related to maternal HIV disclosure. Affective interaction looks at whether health professionals' are treating patient as a person instead of a case [32]. Effective doctor-patient affective interaction involves empathy, which assesses how well the health professionals build up shared feelings and rapport, and show an accurate understanding and acceptance of patients' feelings and expectations [33]. Perceived empathy of health professionals was associated with adoption of various health-related behaviors, such as utilization of health services [34]. We hypothesized that perceived better instrumental and affective doctor-patient interaction would be associated with higher likelihood of maternal HIV disclosure among HIV-infected mothers. Such hypothesis has not been tested in previous studies. Other factors associated with maternal HIV disclosure documented by previous studies included children's age $[9,22,35]$, gender
[35], level of maturity [36, 37], mothers' age [38], social isolation [22], and fear of stigma [39]. We have considered these factors in this study.

We investigated the prevalence of maternal disclosure to at least one living child aged $>5$ years among a sample of HIV-positive mothers having at least one living child of such age. We also investigated two types of factors that may be associated with maternal HIV disclosure: (1) background variables (socio-demographics and disease-related characteristics) and (2) doctor-patient interactions.

\section{Methods}

\section{Participants and Data Collection}

A cross-sectional survey was conducted among HIV-infected women in three Chinese cities (Dehong, Qinzhou and Hezhou) in Guangxi Province from November 2015 to May 2016. Guangxi Province has one of fastest growth of HIV epidemic in China. The major route of HIV transmission in Guangxi has been changing over time. Blood donation/ transfusion used to be the predominant route of HIV transmission in Guangxi before 1996 [40]. Since the unhygienic plasma collection was banned around 1998, the number of new HIV cases attributed to blood donation/transfusion started to decrease (from about 50\% in 1989-1996 to 0\% in 2017), while those attributed to heterosexual transmission has been increasing sharply (from about 10\% in 1989-1996 to $91.28 \%$ in 2017) [40, 41].

Participants were eligible to take part in the study if they were Chinese women aged $\geq 18$ years and received confirmatory HIV diagnosis. In these three cities, governmental antenatal care (ANC) clinics provides HIV treatment and care for HIV-positive women, and there are in total of eight such ANC clinics in Dehong $(n=4)$, Qinzhou $(n=2)$ and Hezhou $(n=2)$. Details of participant recruitment and data collection was described in elsewhere [42]. According the WHO recommendation, children of school age would be the appropriate target for being informed with their parents' HIV status. This study was conducted based on a subsample of 292 HIV-positive mothers who had at least one living child aged $>5$ years. We chose this cut-off age ( $>5$ years old) for children for some reasons. First of all, studies pointed out by school age (6- 8 years old), children's stage of cognitive development allows them understand concepts that assist maternal HIV disclosure, such as the illness and the medication [43, 44]. In addition, all children in China need to attend primary school education at the age of six. Previous studies suggested that when children who were raised by HIV-infected mothers reached school going age ( $\geq 6$ years), they faced more possible vulnerabilities associated with the HIV-positive status of their parents [45, 46]. Maternal HIV 
disclosure may contribute to children's moral and social development at this stage [45, 46]. Many published studies investigating maternal/parental HIV disclosure used the same cut-off age for children [3, 4, 17, 25, 47-50], including those conducted in China [25, 49, 50]. Ethics approval was obtained from the Survey and Behavioral Research Ethics Committee of the Chinese University of Hong Kong (SBRE-18-431).

\section{Measures}

\section{Design of the Questionnaire}

A panel consisting of four epidemiologists and one psychologist was formed to design the questionnaire. Taking into account of the feedbacks of $10 \mathrm{HIV}$-infected women, the questionnaire was finalized by the panel.

\section{Dependent Variable}

Participants were asked whether they had self-disclosed their HIV positive sero-status to at least one living child aged $>5$ years. If the participants had more than one child aged $>5$ years, they were further asked how many living children aged $>5$ years they have disclosed to.

\section{Background Characteristics of the Participants}

Information collected included socio-demographics (e.g. age, ethnicity, education, monthly household income and number of living children) and disease-related characteristics (e.g. time since HIV diagnosis, whether they were on ART, self-reported physical condition, HIV sero-status of regular male sex partners). Regular male sex partner (RP) was defined as their husband or stable boyfriend.

\section{Instrumental Doctor-Patient Interactions}

Three items were used to measure instrumental interactions between ANC health professionals and the participants regarding maternal HIV disclosure to children, they were: (1) "whether health professionals have discussed with you about HIV sero-status disclosure to their children", (2) "whether you have received counseling services regarding HIV sero-status disclosure to children" and (3) "whether you have received support from health professionals when you were making decision whether to disclose HIV sero-status to children" (Response categories: $1=$ no, $2=$ yes, $3=$ not sure).

\section{Affective Doctor-Patient Interactions}

Perceived empathy of ANC health professionals who were providing PMTCT, HIV treatment or care services was measured by using the 10-item CARE Measure. Such scale evaluated participants' perception on relational and communication in consultation $[51,52]$ (response categories: $1=$ poor to $5=$ excellent). The tool was validated in primary care settings in China and showed good psychometric properties (Cronbach's alpha $=0.962$; factor analysis showed a single solution with high item loading ranged from 0.821 to 0.891) [53]. In this study, the Cronbach alpha of this scale was 0.968 . Single factor was identified by exploratory factor analysis, which explained $77.9 \%$ of the total variance.

\section{Statistical Analysis}

Crude odds ratios (cOR) were reported for the univariate associations between background independent variables and the dependent variable (maternal HIV disclosure to at least one living child aged $>5$ years). Significant background variables $(\mathrm{p}<0.05)$ in the univariate analysis were adjusted in the subsequent multiple logistic regression analysis involving variables related to instrumental and affective patientdoctor interactions. Adjusted odds ratios (aOR) with their 95\% confidence intervals (CIs) were obtained respectively. $\mathrm{R}$ version 3.5.0 was used for data analysis.

\section{Results}

\section{Background Characteristics}

Majority of the participants were Han majority ethnicity (89.7\%), married or cohabiting with a man $(81.5 \%)$, had monthly income no more than 2000 RMB (84.2\%) and at least two living children aged over 5 years $(65.1 \%)$. The mean age of the participants was 43.1 (standard deviation $=10.0)$ and $36.6 \%$ had received education of secondary school or above (Table 1).

Regarding disease-related characteristics, the mean duration since receiving HIV diagnosis was 8.5 years (standard deviation $=3.4$ ). Among the participants, $50.3 \%$ reported selling blood as their route of HIV transmission, $86.0 \%$ were on ART and 35.3\% reported their current health conditions were good/very good. Over $90 \%$ (92.4\%) of their RP were aware of their HIV positive sero-status at the time of survey; $54.2 \%$ of them reported their RP were HIV positive (Table 1).

\section{Maternal HIV Disclosure to Living Children Aged Over Five Years}

Among all participants, $45.2 \%$ had self-disclosed their HIV positive sero-status to at least one living child aged $>5$ years. Among HIV-positive mothers having only one living child aged $>5$ years $(n=105), 15.2 \%$ 
Table 1 Associations between background variables and maternal HIV disclosure to at least one child aged over 5 years old among HIV-infected mothers $(\mathrm{N}=292)$

\begin{tabular}{|c|c|c|}
\hline & $\mathrm{N}(\%)$ & Crude OR (95\% CI) \\
\hline \multicolumn{3}{|l|}{ Socio-demographic variables } \\
\hline Age (mean/SD) & $43.1(10.0)$ & $1.21(1.16,1.26)^{* * *}$ \\
\hline \multicolumn{3}{|l|}{ Ethnicity } \\
\hline Han majority & $262(89.7)$ & Ref. \\
\hline Other minorities & $30(10.3)$ & $0.04(0.01,0.26)^{* *}$ \\
\hline \multicolumn{3}{|l|}{ Highest educational level } \\
\hline Primary or below & $185(63.4)$ & Ref. \\
\hline Secondary and above & $107(36.6)$ & $0.18(0.10,0.31)^{* * *}$ \\
\hline \multicolumn{3}{|l|}{ Monthly household income (RMB) } \\
\hline$\leq 1000$ & $147(50.3)$ & Ref. \\
\hline $1001-2000$ & $99(33.9)$ & $0.79(0.77,2.14)$ \\
\hline$>2000$ & $46(15.8)$ & $0.13(0.05,0.32)^{* * *}$ \\
\hline \multicolumn{3}{|l|}{ Number of living children aged over 5} \\
\hline 1 & $105(36.0)$ & Ref. \\
\hline 2 & $116(39.7)$ & $7.10(3.74,13.60)^{* * *}$ \\
\hline 3 & $61(20.9)$ & $15.64(7.10,34.12)^{* * *}$ \\
\hline 4 & $10(3.4)$ & $22.64(4.39,116.75) * * *$ \\
\hline \multicolumn{3}{|l|}{ Diseases-related characteristics } \\
\hline Time since HIV diagnosis (Mean/SD) & $8.5(3.4)$ & $1.58(1.42,1.78)^{* * *}$ \\
\hline \multicolumn{3}{|l|}{ Self-reported route of HIV infection } \\
\hline Sexual transmission & $129(44.2)$ & Ref. \\
\hline Selling blood & $147(50.3)$ & $55.25(6.94,440.16)^{* * *}$ \\
\hline Injective drug use & $2(0.7)$ & - \\
\hline Don't known & $14(4.8)$ & $1.33(0.16,11.07)$ \\
\hline \multicolumn{3}{|l|}{ On antiretroviral treatment } \\
\hline No & $41(14.0)$ & Ref. \\
\hline Yes & $251(86.0)$ & $1.52(1.48,2.97)$ \\
\hline \multicolumn{3}{|l|}{ Self-reported physical condition } \\
\hline Fair & $155(53.1)$ & Ref. \\
\hline Very poor/poor & $34(11.6)$ & $2.59(1.21,5.64) *$ \\
\hline Good/very good & $103(35.3)$ & $0.70(0.69,1.90)$ \\
\hline \multicolumn{3}{|l|}{ HIV status of regular male sex partner } \\
\hline Negative & $112(38.4)$ & Ref. \\
\hline Positive & $155(53.1)$ & $0.49(0.30,1.24)^{* *}$ \\
\hline Unknown/do not have regular male sex partner & $25(8.6)$ & - \\
\hline
\end{tabular}

Regular male sex partner is defined as their husbands or stable boyfriends

Crude OR and $95 \%$ CI for variables with $p<0.05$ in univariate analysis were bold

$O R$ odds ratios; $C I$ confidence interval

$* \mathrm{p}<0.05, * * \mathrm{p}<0.01, * * * \mathrm{p}<0.001$ had self-disclosed to such child. Among those having two living children aged $>5$ years $(n=116)$, the prevalence of maternal HIV disclosure to one of the children and both children were 13.8 and $41.4 \%$, respectively. Among 71 mothers having at least three living children aged $>5$ years, $2.8 \%$ had self-disclosed to some of the children, while $70.4 \%$ had self-disclosed to all children.

\section{Instrumental and Affective Doctor-Patient Interactions}

Since HIV diagnosis, $50.7 \%$ of the participants self-reported that health professionals had discussed with them about maternal HIV disclosure to their children. When struggling on whether to self-disclose HIV sero-status to children, 48.2 
Table 2 Associations between doctor-patient interactions and maternal HIV disclosure to at least one child aged over 5 years old among HIVpositive mothers $(\mathrm{N}=292)$

\begin{tabular}{|c|c|c|c|}
\hline & $\mathrm{N}(\%)$ & Crude OR $(95 \%$ CI) & Adjusted OR (95\% CI) \\
\hline \multicolumn{4}{|l|}{ Instrumental interactions } \\
\hline \multicolumn{4}{|c|}{$\begin{array}{l}\text { Health professionals had discussed with you about disclosure of HIV positive sero- } \\
\text { status to children }\end{array}$} \\
\hline No/not sure & $144(49.3)$ & Ref. & Ref. \\
\hline Yes & $148(50.7)$ & $1.84(1.15,2.94)^{*}$ & $5.85(2.10,16.33)^{* * *}$ \\
\hline \multicolumn{4}{|c|}{$\begin{array}{l}\text { Had received counseling service from health professionals regarding to HIV sero- } \\
\text { status disclosure to children }\end{array}$} \\
\hline No/not sure & $151(51.7)$ & Ref. & Ref. \\
\hline Yes & $141(48.2)$ & $2.08(1.28,3.35)^{* *}$ & $7.84(2.49,24.64) * * *$ \\
\hline \multicolumn{4}{|c|}{$\begin{array}{l}\text { Had received support from health professionals when you were making decision } \\
\text { whether to disclose HIV sero-status to children }\end{array}$} \\
\hline No/not sure & $153(52.4)$ & Ref. & Ref. \\
\hline Yes & 139 (47.6) & $1.82(1.14,2.94)^{*}$ & $8.75(2.70,28.37)^{* * *}$ \\
\hline \multicolumn{4}{|l|}{ Affective interaction } \\
\hline Care measure mean $(\mathrm{SD})^{\mathrm{a}}$ & $33.4(8.8)$ & $1.12(1.08,1.16)^{* * *}$ & $1.09(1.03,1.14) * * *$ \\
\hline
\end{tabular}

Crude OR, adjusted OR, and 95\% CI for variables with $p<0.05$ in univariate/multivariate analysis were bold

$O R$ odds ratios; $C I$ confidence interval

$* \mathrm{p}<0.05, * * \mathrm{p}<0.01, * * * \mathrm{p}<0.001$

${ }^{a}$ CARE Measure: 10 items, Cronbach's alpha $=0.968$, single factor was identified by exploratory factor analysis, which explained $77.9 \%$ of the total variance

and $47.6 \%$ of them received counseling services and support from health professionals (Table 2). Overall, participants gave more positive feedbacks on the empathy shown by the health professionals (mean score of CARE Measure $=33.4$, standard deviation $=8.8$; Table 2 ).

\section{Factors Associated with Maternal HIV Disclosure to at Least One Living Child Aged Over Five Years}

In univariate analysis, older age, having more living children aged $>5$ years, self-reported having poor/very poor physical condition, and self-reported route of HIV infection to be selling blood were more likely to have self-disclosed HIV positive sero-status to at least one living child aged over 5 years. Those reported having secondary education or above, monthly income more than 2000RMB/month, and a HIV-positive RP had lower likelihood of maternal HIV disclosure (cOR and 95\% CI were shown in Table 2).

After adjusting for the aforementioned significant background variables, those self-reported health professionals had discussed with them about maternal HIV disclosure (aOR: 5.85; 95\% CI: 2.10, 16.33), had received counseling services (aOR: 7.84, 95\% CI: $2.49,24.64$ ) or support (aOR: $8.75,95 \%$ CI: $2.70,28.37$ ) when making decision on whether to disclose HIV positive sero-status to children, and perceived higher empathy of health professionals (aOR: $1.09,95 \%$ CI: $1.03,1.14$ ) were more likely to have reported maternal HIV disclosure (Table 2).

\section{Discussion}

To our knowledge, very few studies on maternal HIV disclosure were conducted in China. This study provided some useful information to health workers and policy makers by examining the prevalence of maternal HIV disclosure in a sample of Chinese mothers and the influence of doctorpatient interactions on maternal HIV disclosure. Less than half of the sampled HIV-positive mothers reported maternal HIV disclosure to at least one living child aged $>5$ years. Such prevalence was slightly higher than that reported by a study conducted in central China, where unhygienic plasma collection is the predominant route of HIV transmission [25]. Maternal disclosure can only be beneficial to both children and mothers if it is well-planned and appropriate [25]. According to the WHO, a well-planned and appropriate maternal HIV disclosure to children should consider children's cognitive-developmental ability to understand what is being said. For younger children, they should be informed incrementally to accommodate their cognitive skills and maturity. Mothers should also address children's fear and concerns prior to maternal HIV disclosure [18]. Previous studies conducted in China showed that about $95 \%$ of the undisclosed children knew or were suspicious about their mothers' illness [25]. Although no evidence showed that children whose maternal HIV status was disclosed to them by other sources displayed worse well-being than those children disclosed to by their mothers, studies consistently 
showed that mothers would prefer to take the leading role in disclosing their own HIV status, as they believed they would be the most appropriate person to handle their child's reactions and to facilitate a positive disclosure [54]. Therefore, facilitating maternal HIV disclosure in China is warranted.

Older age and longer time since HIV diagnosis were associated with higher likelihood of maternal HIV disclosure. Such finding was consistent with previous studies showing that delayed parental HIV disclosure was prevalent in China [49]. HIV-infected mothers of other ethnic minorities required more support as they reported much lower prevalence of maternal HIV disclosure. Since disclosure behavior is culturally relevant, future studies should understand whether traditional values/norms of these ethnic minorities would be barriers for maternal HIV disclosure. HIV-infected mothers with better socio-economic status (i.e. higher education and income level) and better self-reported physical condition were less likely to have maternal HIV disclosure. They may not have urgent need to prepare their children for stress events due to their HIV infection (e.g. parent death or financial difficulties of the family). Having a HIV-positive RP was also associated with lower likelihood of maternal HIV disclosure. It is possible that for parents who are both HIV positive, maternal disclosure may involve discussion of father's health condition/illness, which will make the process of disclosure more complicated. HIV-infected mothers having only one living child reported the lowest prevalence of maternal disclosure. It is understandable as disclosing to the children for the first time is always the most difficult. With the experiences and skills learned from their first maternal disclosure, it will be easier for them to disclose to other children. Another possible explanation for this finding may be it is more difficult to conceal their illness when one or more of their children have already known about their illness.

Findings of this study also highlighted the importance of interactions between ANC health professionals and HIVinfected mothers in facilitating maternal HIV disclosure. Due to lack of guideline/policy for health professionals in supporting PLWH in performing the disclosure, only half of HIV-infected mothers reported instrumental doctor-patient interactions (i.e. initiating discussion, provision of counseling and supports related to maternal HIV disclosure) since HIV diagnosis. Having such instrumental doctorpatient interactions was associated with higher maternal HIV disclosure. Therefore, interventions should improve the current practices of these health professionals and there is large room for improvement. It is necessary to include support for maternal HIV disclosure as an essential part of HIV care and management for HIV-infected mothers. Since maternal HIV disclosure is a complex process, it is important to talk about how to disclose rather than whether to disclose. Training should be given to these health professionals. The contents of the training should focus on how to address common concerns related to maternal HIV disclosure, and some practical skills for disclosure (e.g. how to select the timing of disclosure, how to initiate the disclosure process) to better support HIV-infected mothers. In line with our hypothesis, perceived higher empathy of ANC health professionals was also associated with higher maternal disclosure. Future training should also emphasize on how to increase empathy of these health professionals and there is still room for improvement. Interventions targeting health professionals should encourage them to listen to HIV-infected mothers, explore and understand their real concerns, show more compassion and try to be more positive. There were effective interventions in the literatures to increase empathy of health professionals [55]. Since the number of health professionals in ANC would be relatively small, training to improve the situation should be feasible.

This study also had some limitations. First of all, the participants were recruited in cities which were relatively rural and with lower level of income. The findings might not be generalizable to all HIV-infected women in China. Second, response bias to questions related to perceived empathy might exist as participants might have received services from more than one doctor or only from a nurse. Since each HIVinfected woman is followed up by a same case manager since diagnosis and nurses in ANC settings are seldom involved in providing counseling or health education to HIV-infected women, we expected such bias would be small. Third, the maternal HIV disclosure rate may be overestimated, as previous studies suggested that some children were told by parents about their illness in some general terms (e.g. blood diseases) without mentioning HIV/AIDS explicitly [25]. Due to the limited length of the interview, we did not ask about the details about what participants had told their children about their HIV infection. Fourth, we did not ask about whether maternal disclosure was planned or unplanned (e.g. when the mothers were confronted by the children questioning about their illness). Planned and unplanned maternal disclosure may have different implications on the well-being of both HIV-infected mothers and children. Fifth, it was a limitation that the route of HIV transmission was self-reported. Social desirability might occur, some participants would intentionally report their route of HIV transmission as blood donation/transfusion. Studies conducted in Guangxi suggested that people would consider HIV infection through sexual behaviors as a punishment for their lifestyle improprieties, while people infected by medical accidents (e.g. blood transfusion) were considered as "innocent" victims [56]. It was also a limitation that we were not able use the median level for China as the cut-off point to categorize the variable on monthly household income. Furthermore, since the data was collected by face-to-face interview, the interviewer and the participants might know each other. Reporting bias due to social desirability might exist. Computer assisted methods 
could have been used to reduce such bias [57]. Lastly, this was a cross-sectional study and could not establish causal relationships.

\section{Conclusion}

Only $45.2 \%$ of the HIV-infected mothers reported maternal HIV disclosure to at least one living child aged over 5 years. The maternal disclosure rate was extremely low among those only had one living child. Instrumental and affective interactions between ANC health professionals and HIV-infected mothers were important factors in facilitating maternal HIV disclosure. It is necessary to include support for maternal HIV disclosure as an essential part of HIV care and management for HIV-infected mothers. Training should be provided to ANC health professionals to increase their capacity in supporting maternal HIV disclosure and empathy.

\section{Compliance with Ethical Standards}

Conflict of interest The authors declare that they have no conflict of interest.

Research Involving Human Participants All procedures performed in studies involving human participants were in accordance with the ethical standards of the Survey and Behavioural Research Ethics Committee, the Chinese University of Hong Kong and with the 1964 Helsinki declaration and its later amendments or comparable ethical standards.

Informed Consent Informed consent was obtained from all individual participants included in the study.

\section{References}

1. Derlega VJ, Metts S, Petronio S, Margulis ST. Self-disclosure. Newbury Park: Sage; 1993.

2. Krauss BJ, Letteney S, De Baets AJ, Baggaley R, Okero FA. Caregiver's HIV disclosure to children 12 years and under: a review and analysis of the evidence. AIDS Care. 2013;25(4):415-29.

3. Armistead L, Tannenbaum L, Forehand R, Morse E, Morse P. Disclosing HIV status: are mothers telling their children? J Pediatr Psychol. 2001;26(1):11-20.

4. Murphy DA, Armistead L, Marelich WD, Payne DL, Herbeck DM. Pilot trial of a disclosure intervention for HIV + mothers: the TRACK program. J Consult Clin Psychol. 2011;79(2):203-14.

5. Lee MB, Rotheram-Borus MJ. Parents' disclosure of HIV to their children. AIDS. 2002;16(16):2201-7.

6. Letteney S, LaPorte HH. Deconstructing stigma: perceptions of HIV-seropositive mothers and their disclosure to children. Soc Work Health Care. 2004;38(3):105-23.

7. Rotheram-Borus MJ, Draimin BH, Reid HM, Murphy DA. The impact of illness disclosure and custody plans on adolescents whose parents live with AIDS. AIDS. 1997;11(9):1159-64.

8. Schrimashaw EW, Karolynn S. HIV-infected mothers' disclosure to their uninfected children: rates, reasons, and reactions. J Soc Personal Relatsh. 2002;19(1):19-43.
9. Vallerand AH, Hough E, Pittiglio L, Marvicsin D. The process of disclosing HIV serostatus between HIV-positive mothers and their HIV-negative children. AIDS Patient Care STDs. 2005;19(2):100-9.

10. Wiener LS, Battles HB, Heilman NE. Factors associated with parents' decision to disclose their HIV diagnosis to their children. Child Welf. 1998;77(2):115-35.

11. Kennedy DP, Cowgill BO, Bogart LM, Corona R, Ryan GW, Murphy DA, et al. Parents' disclosure of their HIV infection to their children in the context of the family. AIDS Behav. 2010;14(5):1095-105.

12. Conserve DF, Eustache E, Oswald CM, Louis E, King G, Scanlan F, et al. Disclosure and impact of maternal HIV + serostatus on mothers and children in rural Haiti. Matern Child Health J. 2014;18(10):2309-15.

13. Murphy DA. HIV-positive mothers' disclosure of their serostatus to their young children: a review. Clin Child Psychol Psychiatry. 2008;13(1):105-22.

14. Conserve DF, King G. An examination of the HIV serostatus disclosure process among Haitian immigrants in New York City. AIDS Care. 2014;26(10):1270-4.

15. Asander AS, Bjorkman A, Belfrage E, Faxelid E. HIV-infected African parents living in Stockholm, Sweden: disclosure and planning for their children's future. Health Soc Work. 2009;34(2):107-15.

16. Murphy DA, Marelich WD, Hoffman D. A longitudinal study of the impact on young children of maternal HIV serostatus disclosure. Clin Child Psychol Psychiatry. 2002;7(1):55-70.

17. Murphy DA, Steers WN, Dello Stritto ME. Maternal disclosure of mothers' HIV serostatus to their young children. J Fam Psychol. 2001;15(3):441-50.

18. World Health Organization. Guideline on HIV disclosure counselling for children up to 12 years of age. 2011.

19. Hawk ST. Disclosures of maternal HIV infection to seronegative children: a literature review. J Soc Personal Relatsh. 2007;24(5):657-73.

20. Li X, de Wit J, Qiao S, Sherr L. HIV disclosure to children in low-and middle-income countries: towards effective interventions. AIDS. 2015;29(Suppl 1):S1-5.

21. Murphy DA, Roberts KJ, Hoffman D. Young children's reactions to mothers' disclosure of maternal HIV + serostatus. J Child Fam Stud. 2006;15(1):38-55.

22. Corona R, Beckett MK, Cowgill BO, Elliott MN, Murphy DA, Zhou AJ, et al. Do children know their parent's HIV status? parental reports of child awareness in a nationally representative sample. Ambul Pediatr. 2006;6(3):138-44.

23. Palin FL, Armistead L, Clayton A, Ketchen B, Lindner G, KokotLouw P, et al. Disclosure of maternal HIV-infection in South Africa: description and relationship to child functioning. AIDS Behav. 2009;13(6):1241-52.

24. Qiao S, Li X, Stanton B. Disclosure of parental HIV infection to children: a systematic review of global literature. AIDS Behav. 2013;17(1):369-89.

25. Zhao J, Li X, Qiao S, Zhao G, Zhang L, Stanton B. Parental HIV disclosure: from perspectives of children affected by HIV in Henan China. AIDS Care. 2015;27(4):416-23.

26. Ministry of Health People's Republic of China, Joint United Nations Programme on HIV/AIDS, World Health Organization. 2011 estimates for the HIV/AIDS epidemic in China. Beijing: 2011.

27. Tse CY, Chong A, Fok SY. Breaking bad news: a Chinese perspective. Palliat Med. 2003;17(4):339-43.

28. Spangler SA, Onono M, Bukusi EA, Cohen CR, Turan JM. HIVpositive status disclosure and use of essential PMTCT and maternal health services in rural Kenya. J Acquir Immune Defic Syndr. 2014;67(Suppl 4):S235-42. 
29. Rochat TJ, Mkwanazi N, Bland R. Maternal HIV disclosure to HIV-uninfected children in rural South Africa: a pilot study of a family-based intervention. BMC Pub Health. 2013;13:147.

30. Simoni JM, Yang JP, Shiu CS, Chen WT, Udell W, Bao M, et al. Nurse-delivered counselling intervention for parental HIV disclosure: results from a pilot randomized controlled trial in China. AIDS. 2015;29(Suppl 1):S99-107.

31. Ben-Sira Z. The function of the professional's affective behavior in client satisfaction: a revised approach to social interaction theory. J Health Soc Behav. 1976;17(1):3-11.

32. Ben-Sira Z. Affective and instrumental components in the physician-patient relationship: an additional dimension of interaction theory. J Health Soc Behav. 1980;21(2):170-80.

33. Norfolk T, Birdi K, Walsh D. The role of empathy in establishing rapport in the consultation: a new model. Med Educ. 2007;41(7):690-7.

34. van Ryn M, Fu SS. Paved with good intentions: do public health and human service providers contribute to racial/ethnic disparities in health? Am J Pub Health. 2003;93(2):248-55.

35. Armistead L, Tannenbaum L, Forehand R, Morse E, Morse P. Disclosing HIV Status: are mothers telling their children? J Pediatr Psychol. 2001;26(1):11-20.

36. Delaney RO, Serovich JM, Lim JY. Reasons for and against maternal HIV disclosure to children and perceived child reaction. AIDS care. 2008;20(7):876-80.

37. Vreeman RC, Gramelspacher AM, Gisore PO, Scanlon ML, Nyandiko WM. Disclosure of HIV status to children in resource-limited settings: a systematic review. J Int AIDS Soc. 2013;16(1):18466.

38. Serovich JM. A test of two HIV disclosure theories. AIDS Educ Prev. 2001;13(4):355-64.

39. Ostrom RA, Serovich JM, Lim JY, Mason TL. The role of stigma in reasons for HIV disclosure and non-disclosure to children. AIDS Care. 2006;18(1):60-5.

40. The World Bank. China: the epidemiological and behavioral dynamos of the HIV epidemic in Guangxi Province. Available at: http://documents.worldbank.org/curated/en/741471468261298 390/pdf/686700ESW0P0750mic0Guangxi0Province.pdf. 2006. Accessed 15 May 2019.

41. Ge XM, Yang WM, Zhu QY, Wu XL, Shen ZY, Zhu JH, et al. [Epidemiological characteristics of HIV/AIDS in Guangxi Zhuang Autonomous Region, 2010-2017]. Zhonghua Liu Xing Bing Xue Za Zhi=Zhonghua liuxingbingxue zazhi. 2019;40(3):315-21.

42. Wang Z, Lau JT, Wang Q, Mo P. Prevalence of and factors associated with inconsistent condom use with HIV discordant male regular sex partners among HIV-infected women in China. J Sex Marital Ther. 2019;45(4):1-11.

43. Bibace R, Walsh ME. Development of children's concepts of illness. Pediatrics. 1980;66(6):912-7.

44. Lesch A, Swartz L, Kagee A, Moodley K, Kafaar Z, Myer L, et al. Paediatric HIV/AIDS disclosure: towards a developmental and process-oriented approach. AIDS Care. 2007;19(6):811-6.

45. Bauman LJC. Behavioural problems in school-aged children of mothers with HIV/AIDS. Clin Child Psychol Psychiatry. 2002;7(39):39-54.
46. McNally LM, Hadingham J, Archary D, Moodley R, Coovadia HM. HIV-exposed but uninfected children: why are they vulnerable? Vulnerable Child Youth Stud. 2006;1(2):139-48.

47. Shaffer A, Jones DJ, Kotchick BA, Forehand R. Telling the children: disclosure of maternal HIV infection and its effects on child psychosocial adjustment. J Child Fam Stud. 2001;10(3):301-13.

48. Armistead L, Morse E, Forehand R, Morse P, Clark L. AfricanAmerican women and self-disclosure of HIV infection: rates, predictors, and relationship to depressive symptomatology. AIDS Behav. 1999;3(3):195-204.

49. Mao Y, Li X, Qiao S, Zhao Q, Zhou Y, Shen Z. Social support, stigma, and HIV disclosure among parents living with HIV in Guangxi, China. AIDS Care. 2018;30(2):168-72.

50. Li H, Li X, Tso LS, Qiao S, Holroyd E, Zhou Y, et al. HIV-negative children's experiences and opinions towards parental HIV disclosure: a qualitative study in China. Vulnerable Child Youth Stud. 2016;11(2):173-9.

51. Mercer SW, McConnachie A, Maxwell M, Heaney D, Watt GC. Relevance and practical use of the consultation and relational empathy (CARE) measure in general practice. Fam Pract. 2005;22(3):328-34.

52. Mercer SW, Maxwell M, Heaney D, Watt GC. The consultation and relational empathy (CARE) measure: development and preliminary validation and reliability of an empathy-based consultation process measure. Fam Pract. 2004;21(6):699-705.

53. Fung CS, Hua A, Tam L, Mercer SW. Reliability and validity of the Chinese version of the CARE Measure in a primary care setting in Hong Kong. Fam Pract. 2009;26(5):398-406.

54. Delaney RO, Serovich JM, Lim JY. Psychological differences between HIV-positive mothers who disclose to all, some, or none of their biological children. J Marital Fam Ther. 2009;35(2): 175-80.

55. Altiner A, Brockmann S, Sielk M, Wilm S, Wegscheider K, Abholz HH. Reducing antibiotic prescriptions for acute cough by motivating GPs to change their attitudes to communication and empowering patients: a cluster-randomized intervention study. J Antimicrob Chemother. 2007;60(3):638-44.

56. Zhang C, Li X, Liu Y, Qiao S, Zhang L, Zhou Y, et al. Stigma against people living with HIV/AIDS in China: does the route of infection matter? PLoS ONE. 2016;11(3):e0151078.

57. Wang Z, Wu X, Lau J, Mo P, Mak W, Wang X, et al. Prevalence of and factors associated with unprotected anal intercourse with regular and nonregular male sexual partners among newly diagnosed HIV-positive men who have sex with men in China. HIV Med. 2017;18(9):635-46.

Publisher's Note Springer Nature remains neutral with regard to jurisdictional claims in published maps and institutional affiliations. 